\title{
FRECUENCIA Y SEVERIDAD DE ENFERMEDAD PERIODONTAL EN PACIENTES CANINOS DE UNA CLÍNICA DE ANIMALES MENORES EN LIMA
}

\section{Frequency and Severity of Periodontal Disease in Canine Patients of a Pet Clinic in Lima City}

\author{
Arturo Maetahara R. ${ }^{1}$, Viviana Fernández P. ${ }^{1,2}$, Ysaac Chipayo G. ${ }^{1}$, \\ Francisco Suárez A. ${ }^{3}$
}

\section{Resumen}

\begin{abstract}
Se evaluó la frecuencia de presentación de enfermedad periodontal en 52 caninos mayores del año de edad, pacientes de la Clínica de Animales Menores de la Facultad de Medicina Veterinaria de la Universidad Nacional Mayor de San Marcos, entre octubre de 2005 a marzo de 2006, a través del Índice Veterinario Periodontal. Se encontró una frecuencia de $76.9 \%$ (40/52) de enfermedad periodontal, correspondiendo el $48.1 \%(25 / 52)$ a gingivitis, $21.2 \%(11 / 52)$ a periodontitis leve y $7.7 \%$ (4/52) a periodontitis moderada. Los dientes más afectados fueron los de la región maxilar y los premolares. Se encontró una asociación positiva entre edad con la frecuencia y severidad de la enfermedad periodontal. Asimismo, se observó una mayor pérdida de dientes en los perros de mayor edad.
\end{abstract}

Palabras clave: enfermedad periodontal, perros, gingivitis, periodontitis

\section{Abstract}

The frequency of periodontal disease was evaluated in 52 canines older than one year of age, patients of the Small Animal Clinic, School of Veterinary Medicine of San Marcos University, Lima, during the period of October 2005 to March 2006. The veterinary periodontal index was used. The results showed a frequency of $76.9 \%(40 / 52)$ of dogs with periodontal disease, where $48.1 \%$ (25/52) had gingivitis, $21.2 \%$ (11/52) mild periodontitis, and $7.7 \%(4 / 52)$ moderate periodontitis. The more affected type of teeth was the premolars and the superior teeth. There was a positive association between age with frequency and severity of periodontal disease. It was also observed that missing teeth was higher in older dogs.

Key words: periodontal disease, dogs, gingivitis, periodontitis

\footnotetext{
${ }^{1}$ Clínica de Animales Menores, ${ }^{3}$ Laboratorio de Medicina Veterinaria Preventiva, Facultad de Medicina Veterinaria, Universidad Nacional Mayor de San Marcos, Lima

${ }^{2}$ E-mail: viviferpar@hotmail.com
} 


\section{INTRODUCCIÓN}

La enfermedad periodontal (EP) se define como la respuesta inflamatoria a la placa dental (Gorrel, 1998), y es la patología oral más común en perros y gatos adultos (Hamp et al., 1984; Reichart et al., 1984). El término periodonto se refiere a los tejidos que rodean y soportan al diente (Harvey y Emily, 1993), y se compone de la encía, cemento, ligamento periodontal y hueso alveolar, y cuya función principal es unir los dientes al tejido óseo de los maxilares.

La EP se compone de dos procesos: la gingivitis (proceso reversible) y la periodontitis que, además de gingivitis, incluye una pérdida variable del tejido de soporte del diente. Este proceso es la causa más importante de pérdidas de piezas dentales tanto en humanos como en animales de compañía (Colmery y Frost, 1986).

Diversos estudios han reportado una alta frecuencia de EP en perros, que han permitido conocer el rol de la placa dental y de otros factores asociados, como edad, tamaño del animal, biotipo cefálico, dieta y comportamiento masticatorio (Tangsiri y Emami, 2003). La EP afecta la salud sistémica, pues existe una correlación positiva entre la severidad de la EP y cambios histológicos observados en corazón, pulmones, riñón e hígado de perros (Debowes et al., 1996).

El presente estudio tuvo por objetivo poder determinar la frecuencia y severidad de presentación de la enfermedad periodontal en pacientes caninos de una clínica de animales menores de Lima.

\section{Materiales y Métodos}

Se empleó 52 caninos mayores de un año de edad, sin distinción de raza y sexo, que fueron pacientes de la Clínica de Ani- males Menores de la Facultad de Medicina Veterinaria de la Universidad Nacional Mayor de San Marcos (FMV-UNMSM), Lima, entre el 1 de octubre de 2005 al 30 de marzo de 2006. Los canes procedieron de diferentes distritos de Lima Metropolitana y acudieron a la clínica por motivos diferentes al estudio, como consultas, tratamientos, cirugías, etc.

Los animales fueron manejados bajo anestesia, usando diazepam $(0.25 \mathrm{mg} / \mathrm{kg}$, vía intravenosa) como premedicación, clorhidrato de ketamina $(7.5-10 \mathrm{mg} / \mathrm{kg}$, vía endovenosa) para inducción y mantenidos con anestesia inhalatoria de sevoflurano durante todo el procedimiento diagnóstico.

Se evaluó el número de dientes presentes. La fórmula dentaria estándar de la dentición permanente usada fue de I3/3, C1/1, P4/ 4, M2/3 (Page y Schroeder, 1976). Con la sonda periodontal se revisó la presencia de recesión gingival o hiperplasia gingival y la profundidad del surco gingival. Se registró, además, el sangrado al sondaje, el grado de movilidad dental y la afección de furca en dientes multirradiculares, según Wiggs y Lobprise (1997). Al final de cada examen, se otorgó un puntaje a cada pieza dentaria de acuerdo al Índice Veterinario Periodontal (IVP) que considera los siguientes valores (Wiggs y Lobprise, 1997):

0: No hay signos de gingivitis ni de periodontitis.

1: Gingivitis. Inflamación de la encía y sangrado al sondaje, pero no hay pérdida de unión.

2: Periodontitis leve. Lo anterior, más los signos iniciales de periodontitis destructiva. Bolsa periodontal de 3-5 mm, pérdida de unión de 0-2 $\mathrm{mm}$, movilidad dental grado 0 ó 1 , y un grado de afección de furca 0 ó 1 .

3: Periodontitis moderada. Bolsa periodontal de 5-7 mm, pérdida de unión de 2-4 mm, movilidad dental grado 1 ó 2 y grado de afección de furca 1 ó 2. 
4: Periodontitis severa. Bolsa periodontal con más de $7 \mathrm{~mm}$, pérdida de unión mayor a $4 \mathrm{~mm}$, movilidad del diente grado 2 ó 3 y grado de afección de furca 2 ó 3.

El promedio de los valores resultantes de todas las piezas dentarias presentes de cada animal es el IVP y los valores se interpretan como normal o sano $(<0.1)$, gingivitis $(0.1-1.0)$, periodontitis leve $(1.1-2.0)$, periodontitis moderada $(2.1-3.0) \mathrm{y}$ periodontitis severa $(3.1-4.0)$.

Se determinó la frecuencia porcentual de las afecciones periodontales, y se analizaron usando la prueba de Chi Cuadrado.

\section{Resultados}

El 76.9\% (40/52) de caninos presentaron algún grado de enfermedad periodontal. Se observó un incremento en la frecuencia y severidad de la enfermedad periodontal con relación a la edad (Cuadro 1). En el Cuadro 2 se observa que la frecuencia de canes con mayor número de piezas dentarias faltantes aumenta con la edad.

El tipo dentario más frecuentemente afectado fue los premolares (58.9\%), seguido de los incisivos (31.2\%), molares (28.6\%) y caninos $(26.3 \%)(\mathrm{p}<0.05)$. Los dientes superiores fueron más afectados que los inferiores (37.8 vs. $30.0 \%, \mathrm{p}<0.05)$.

\section{Discusión}

La frecuencia de enfermedad periodontal $(76.9 \%, 40 / 52)$ del presente estudio fue similar a resultados encontrados en otros países (Hamp et al., 1984; Isogai et al., 1989; Kyllar y Witter, 2005). Sin embargo, hay evidencias de frecuencias mayores, como el $97 \%$ reportado por Gad (1968) y el $90 \%$ hallado por Hoffmann y Gaengler (1996) en perros de la raza Poodle, posiblemente debido al pequeño tamaño del cráneo. No obstante lo anterior, la frecuencia encontrada en el presente estudio se considera alta y podría deberse a que la mayoría de profesionales veterinarios no evalúan adecuadamente la cavidad oral, pues posiblemente subestimen las consecuencias de esta enfermedad sobre la salud integral del paciente, aspecto que ha sido reconocido en diversos estudios (Debowes et al., 1996; Pavlica, 2002). Además, en el país, las medidas de prevención, dirigidas al control de la placa y sarro dental, y los tratamientos periodontales (medidas dirigidas a la conservación de los dientes afectados) no son aplicadas regularmente en las mascotas.

La relación entre edad con la frecuencia y severidad de la EP encontrada en el presente trabajo es consistente con otros estudios (Hamp et al., 1984; Isogai et al., 1989; Harvey et al., 1994; Hoffman y Gaengler, 1996; Kyllar y Witter, 2005), pese a que estas diferencias no fueron estadísticamente significativas, posiblemente debido al pequeño tamaño muestral. El efecto acumulativo de la placa dental a lo largo del tiempo es el responsable de la presentación de la enfermedad, ya que los perros, normalmente, no reciben higiene dental. Se menciona, también, que puede estar directamente asociada a procesos de envejecimiento de los tejidos periodontales, tanto en su estructura como en el proceso de curación (Anderson, 1982), dado que las defensas del organismo de un animal mayor no resultan tan eficaces en el control de la enfermedad como en un animal más joven.

La pérdida de dientes puede ocurrir como consecuencia de una EP severa en la mayoría de los casos (Harvey et al., 1994; Kyllar y Witter, 2005). En el presente estudio, el número de animales con dientes faltantes así como el promedio de dientes faltantes por animal se incrementó con la edad. La causa de la pérdida dentaria puede deberse a causas ajenas a la EP (agénesis dental, trauma, etc.), pero con la historia de una mayor severidad de la EP con la edad, es muy posible que la causa principal en el 
Cuadro 1. Frecuencia y severidad de enfermedad periodontal (según Índice Veterinario Periodontal $^{1}$ ) en pacientes caninos de la Clínica de Animales Menores de la FMV-UNMSM, según el grupo etáreo (octubre 2005 - marzo 2006)

\begin{tabular}{|c|c|c|c|c|c|c|c|c|c|}
\hline \multirow{3}{*}{$\begin{array}{l}\text { Grupo etáreo } \\
\text { (años) }\end{array}$} & \multicolumn{8}{|c|}{ Enfermedad periodontal } & \multirow{3}{*}{$\begin{array}{l}\text { Total } \\
\left(\mathrm{N}^{\circ}\right)\end{array}$} \\
\hline & \multicolumn{2}{|c|}{ Sin EP } & \multicolumn{2}{|c|}{ Gingivitis } & \multicolumn{2}{|c|}{ P. Leve } & \multicolumn{2}{|c|}{ P. Moderada } & \\
\hline & $\mathrm{n}$ & $\%$ & $\mathrm{n}$ & $\%$ & $\mathrm{n}$ & $\%$ & $\mathrm{n}$ & $\%$ & \\
\hline 1 a 4 & 10 & 58.8 & 6 & 35.3 & 1 & 5.9 & 0 & 0 & 17 \\
\hline 5 a 8 & 1 & 6.3 & 8 & 50.0 & 6 & 37.5 & 1 & 6.3 & 16 \\
\hline Más de 8 & 1 & 5.3 & 11 & 57.9 & 4 & 21.1 & 3 & 15.8 & 19 \\
\hline Total & 12 & 23.1 & 25 & 48.1 & 11 & 21.2 & 4 & 7.7 & 52 \\
\hline
\end{tabular}

Cuadro 2. Frecuencia de pacientes caninos con piezas dentarias faltantes de la Clínica de Animales Menores de la FMV-UNMSM (octubre 2005 - marzo 2006)

\begin{tabular}{|c|c|c|c|c|c|c|c|c|c|}
\hline \multirow{3}{*}{$\begin{array}{l}\text { Grupo etáreo } \\
\text { (años) }\end{array}$} & \multicolumn{8}{|c|}{ Piezas dentarias faltantes } & \multirow{3}{*}{$\begin{array}{r}\text { Tota } \\
\left(\mathrm{N}^{\circ}\right)\end{array}$} \\
\hline & \multicolumn{2}{|c|}{0} & \multicolumn{2}{|c|}{$1-8$} & \multicolumn{2}{|c|}{$9-16$} & \multicolumn{2}{|c|}{$17-24$} & \\
\hline & $\mathrm{n}$ & $\%$ & $\mathrm{n}$ & $\%$ & $\mathrm{n}$ & $\%$ & $\mathrm{n}$ & $\%$ & \\
\hline $1 \mathrm{a} 4$ & 9 & 52.9 & 5 & 29.4 & 2 & 11.8 & 1 & 5.9 & 17 \\
\hline 5 a 8 & 5 & 31.3 & 7 & 43.8 & 3 & 18.8 & 1 & 6.3 & 16 \\
\hline Más de 8 & 4 & 21.1 & 11 & 57.9 & 1 & 5.3 & 3 & 15.8 & 19 \\
\hline Total & 18 & 34.6 & 23 & 44.2 & 6 & 11.5 & 5 & 9.6 & 52 \\
\hline
\end{tabular}

presente estudio haya sido por el desarrollo de periodontitis severa.

Los dientes más frecuentemente afectados fueron los premolares, hallazgo que corrobora otros estudios similares (Kyllar y Witter, 2005). Cuando el perro no recibe una higiene dental frecuente, los dientes, y en especial los premolares, acumulan placa y cálculos dentales debido a la desembocadura de los conductos de las glándulas salivales parótidas y cigomáticas entre el cuarto premolar y primer molar superior, que favorece una deposición mineral constante para la formación rápida de sarro dental (Gioso, 2004). La auto-limpieza dada por el tipo de oclusión, la fuerza mecánica de la masticación, el flujo salival y el movimiento de la lengua resultan ineficientes en la remoción de placa y sarro dental en este tipo dentario (Flores, 2006). En las razas braquicefálicas se observa una rotación de los premolares, lo que permite la creación de un mayor número de potenciales superficies retentivas de placa y sarro dental (Colmery y Frost, 1986).

Kyllar y Witter (2005) señalan que los dientes superiores son más afectados, mientras que otros investigadores (Lindhe et al., 1975; Harvey et al., 1994; Hoffmann y Gaengler, 1996) no encontraron diferencias entre dientes superiores e inferiores. En el presente estudio, los dientes superiores resultaron los más afectados. 


\section{Conclusiones}

? $\quad$ El $76.9 \%$ de caninos mayores de un año de edad presentaron enfermedad periodontal.

? La frecuencia y severidad de la enfermedad periodontal se incrementó con la edad.

? El número de animales con dientes faltantes y el número de dientes faltantes por animal se incrementó con la edad.

? Los dientes superiores y los premolares fueron las piezas dentarias más afectadas.

\section{Literatura Citada}

1. Anderson D. 1982. Periodontal disease and aging. Geriodontology 1(1): 19-23.

2. Colmery B, Frost P, 1986. Periodontal disease: etiology and pathogenesis. Vet Clin N Am Small 16(5): 817-833.

3. Debowes LJ, Mosier D, Logan E, Harvey C, Lowry S, Richardson DC. 1996. Association of periodontal disease and histologic lesions in multiple organs from 45 dogs. J Vet Dent 2: 57-60.

4. Flores E. 2006. Odontología. [Internet], [10 abril 2006]. Disponible en: http:// www.rawmeatybones.com/translations/ spanish/chapter_7_sp.pdf

5. Gad T. 1968. Periodontal disease in dogs. I. Clinical investigation. J Period Res 3: 268-272.

6. Gioso M. 2004. Curso básico: Odontología veterinaria en pequeños animales. Lima: Universidad Peruana Cayetano Heredia.

7. Gorrel C. 1998. Periodontal disease and diet in domestic dogs. J Nutr 128(12): 2712S-2714S.

8. Hamp S, Olsson S, Farso-Madsen K, Viklands P, Fornell J. 1984. A macroscopic and radiologic investigation of dental diseases of the dog. Vet Radiology 25(2): 86-92.
9. Harvey CE, Emily PP. 1993. Periodontal disease. In: Harvey CE; Emily PP (eds). Small animal dentistry. St. Louis: Mosby. p 89-144.

10. Harvey C, Shofer F, Laster L. 1994. Association of age and body weight with periodontal disease in North American dogs. J Vet Dent 11: 94-105.

11. Hoffman T, Gaengler P. 1996. Epidemiology of periodontal disease in poodles. J Small Anim Pract 37: 309-316.

12. Isogai H, Isogai E, Okamoto H, Shirakawa $H$, Nakamura $F$, Matsumoto T, Watanabe T, et al 1989. Epidemiological study on periodontal diseases and some other dental disorders in dogs. Jpn J Vet Sci 51: 1151-1162.

13. Kyllar M, Witter K. 2005. Prevalence of dental disorders in pet dogs. Vet Med Czech 50: 496-505.

14. Lindhe J, Hamp S, Löe H. 1975. Plaque induced periodontal disease in beagle dogs: A 4-year clinical, roentgenographical and histometrical study. J Period Res 10: 243-255.

15. Page R, Schroeder H. 1976. Pathogenesis of chronic inflammatory periodontal disease, a summary of current work. Lab Invest 33: 235-249.

16. Pavlica Z. 2002. Enfermedad periodontal y sus efectos a nivel sistémico en la población canina de riesgo. En: Simposio "Control clínico y nutricional de perros y gatos senior". Granada, España.

17. Reichart P, Durr U, Triadan $H$, Vickendey G. 1984. Periodontal disease in the domestic cat: a histopathologic study. J Period Res 19: 67-75.

18. Tangsiri L, Emami E. 2003. Periodontal disease and the treatments in dogs. [Internet]. [4 June 2005]. Available in: http://www.ki.se/odont/ cariologi_endodonti / 98 b/ LalehTangsiri,EmmaEmami.pdf

19. Wiggs RB, Lobprise HB. 1997. Periodontology. In: Wiggs RB, Lobprise HB (eds). Veterinary dentistry, principles $\&$ practice. Philadelphia: LippincottRaven. p 186-231. 